\title{
Uber das Wachstum und Aussehen des BCG auf dem Kartoffelnährboden mit Rindergalle und gallensaurem Salz.
}

\author{
Von \\ Iwao Kanno. \\ (营 野 䇴) \\ (Aus der Medizinischen Klinik der Kaiserlichen. Tohoku-Universität \\ zu Sendai. Aus dem Institut zur Forschung Säurefester Bazillen \\ an der Kaiserlichen Tohoku-Universität zu Sendai. \\ Leiter: Prof. Dr. T. Kumagai und Prof. Dr. T. Ebina.)
}

\section{Einleitung.}

Über die Variabilitätserscheinungen säurefester Bazillen, insbesondere der Froschbazillen haben schon 1913 Baerthlein und Toyodal) Untersuchungen angestellt und ihre Ergebnisse veröffentlicht, dass diese Bazillen in zweierlei Kolonieformen wachsen : in trockene, bröckelige, blumenkohlartig wuchernde Kolonien, die sich überhaupt nicht verreiben lassen, und in feuchte glänzende mit glatter Oberfläche.

1921 hat auch Gildmeiste ${ }^{2}$ ) bei den Schildkrötenbazillen beiderlei Kolonieformen festgestellt. Später gelang es Petroff, Brauch und Steen $\mathrm{ken}^{3-6)}$ die Tuberkelbazillen insbesondere die abgeschwächten Rinder-

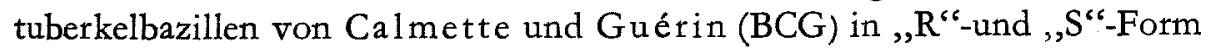
zu dissozieren. Sie haben die biologischen Eigenschaften beider Formen genau untersucht und behaupteten, dass die S-Form des BCG stark virulent ist und bei Meerschweinchen progressive tuberkulöse Veränderungen hervorruft, während die R-Form schwach virulent und ein undissozierter Stammbazillus ist. Kra us und Gerla $\mathrm{ch}^{7)-8}$ ) haben die Mitteilung Petroffs nachgeprüft und berichtet, dass, wenn ihnen auch die Dissoziation der S-Form und R-Form aus BCG gelang, die erstere bei Meerschweinchen keine progressiven tuberkulösen Veränderungen herbeiführen konnte. Auch Tschechnowitzer $^{\text {9) }}$ hat zwei Typen von S- und R-Kolonie aus BCG getrennt, aber er konnte ihre Pathogenität nicht bestätigen. Im Streit über die starke Virulenz der S-Form schien das bekannte schreckliche Ereignis, das 1930 in Lübeck 
geschah, einen festen Anhaltspunkt denjenigen gegeben zu haben, die die beibehaltene starke Virulenz des BCG behaupteten, aber durch die Untersuchungen von $\mathrm{B}$. Lang $\mathrm{e}^{10}$ ) und L. Lange $u$. a. gelangte man zu einem Schluss, dass man in Lübeck den Säuglingen anstatt BCG per os aus Versehen virulente Tuberkelbazillen gegeben hat; die verabreichten Tuberkelbazillen nämlich waren nicht dieselben wie die BCG vom Pasteur-Institut. Birkh a u g ${ }^{11)}$ teilte mit, dass S-Form und R-Form der Tuberkelbazillen von Typus bovinus im Körper der Meerschweinchen ineinander übergehen können. Er berichtete ferner auch über das Vorhandensein der S-Form und R-Form von BCG. Roulet ${ }^{12)}$ züchtete die S-Form des BCG auf Glyzerinkartoffeln mit Zusatz von Rindergalle und fand, dass sich eine Virulenzzunahme der S-Form nicht feststellen liess. Auch die Untersuchungen von Birkhaug ${ }^{13)}$ und Tog un ow a ${ }^{14)}$ führten zu ähnlichen Ergebnissen. Toda ${ }^{15)}$ hat 1931 R-Form und S-Form von Tuberkelbazillen von Typus humanus und BCG getrennt und meinte, dass aus BCG gezüchte , $R^{\prime \prime}$-und ,,$S^{\prime c}$-Kolonie eine fast gleichgradige Virulenz gegen Kaninchen und Meerschweinchen zeigen. Auch Urabe ${ }^{16)}$ veröffentlichte 1936 eine Mitteilung über die Untersuchung betreffs der S-Form und R-Form säurefester Bazillen. Da entsteht nun selbstverständlich eine interessante Frage, was das veranlassende Moment zur Dissoziation der Tuberkelbazillen sei. In diesbezüglicher Literatur liegen die Angaben von Petroff und Mitarbeitern, ${ }^{34(5) 68)}$ Schaefer, ${ }^{17)}$ Batglows ki, ${ }^{18)}$ Smithburn, ${ }^{19)}$ Oatway und Steeken, ${ }^{20 ;}$ Toda, ${ }^{15) 21)}$ Urabe, ${ }^{16)}$ Otomo ${ }^{22)}$ u.a. vor. Als wichtige Veranlassungsmomente sind nach ihnen physikalische Momente, z. B. Veränderungen der Feuchtigkeit im Röhrchen des Nährbodens und der Kulturtemperatur und chemische Momente, vor allem der Eirfluss von $\mathrm{Ph}$, zu nennen. Sie behaupten aber, dass die Dissoziation ausserdem durch Tierpassage, Wechsel des Nährbodens oder zuweilen durch eine Mutation in der abgelaufenen Kultur ohne besondere Ursache geschehen kann. Wenn man BCG auf Glyzerinkartoffeln mit Rindergallezusatz züchtet, so sieht man im allgemeinen eine dicke lehmartige feuchte Kolonie und erkennt die sog. S-Form von Petroff. Es ist aber eine erfahrene Tatsache, dass das Aussehen und Wachstum der S-Form je nach dem Umstande sehr verschieden ist. Die vorliegende Untersuchung wurde durchgeführt,um den Einfluss des Zusatzes von Galle zu verfolgen. Unter Anwendung von Rindergalle und zwei gallensauren Salzarten, Natrium glycocholicum und Natrium taurocholicum, Hauptbestandteilen der Galle, haben wir zu diesem Zwecke BCG auf Kartoffelnährboden mit Zusatz von ihnen gezüchtet und dessen Wachstum und Dissoziation, insbesondere die Etscheinung der SForm beobachtet. Die Versuchsergebnisse seien im Folgenden kurz mitgeteilt. 


\section{Versuchsmethode}

\section{1) Gebraucbte Båillenstämme.}

Zum Versuchen wurden folgende, auf verschiedenen Nährboden dauernd kultivierte BCG-Stämme gebraucht.

a) 1. Bazillenstamm: Dieser BCG-Stumm wurde uns im August 1940 von Prof. Imamura an der Kaiserlichen Universität Osaka gegeben. Er wurde seit Dezember 1927 immer auf 5\% Glyzerinkartoffeln mit Zusatz von Rindergalle und auch in unserer Klinik auf gleichem Nährboden in Intervallen von ungefähr 3 Wochen neu gezüchtet und immer auf diese Weise bis heute kultiviert.

b) 2. Bazillenstamm: Dieser BCG-Stamm wurde uns im August 1940 von dem Institut zur Forschung der Infektionskrakheiten an der Kaiserlichen Universität Tokyo gegeben. Er wurde in dem Institut monatlich auf $5 \%$ Glyzerinbouillonkartoffeln und auch in unserer Klinik auf gleichem Nährboden in Intervallen von ungefähr 3 Wochen neu gezüchtet.

\section{2) Gebraucbte Näbrboden.}

Mittelst Roux's Reagenzgläser wurden 5\% glyzerinhaltige und glyzerinfreie Kartoffelnährböden mit Zusatz von Rindergalle, Natr. glycochol. und Natr. taurochol. nach der Methode von Calmette ${ }^{23)}$ steril hergestellt. Zur Anwendung kamen 2 Arten Rindergalle : die frische, welche nicht über 5 Tage alt war, und die alte, welche nach der Entnahme über 1 Monat stehen gelassen wurde. Sie wurden dann in Verhältnissen von 100, 50, 25, 10, 5 usw. : 100 verdünnt. Gallensaure Salze wurden in Verdünnungen 4-0,05\% benutzt. Natr. glycochol. und Natr. taurochol. von Merck, Deutschland, wurden gebraucht. Auf der Oberfläche der Nährböden wurde jede Art beider, oben erwähnter Bazillenstämme mit einerÖse gleichmässig eingerieben und bei $38^{\circ} \mathrm{C}$ gehalten. Wir haben dann wöchentlich bis 5 Wochen das Wachstum und Aussehen des BCG beobachtet. Das Wachstum (W.) und Aussehen (A.) wurden mit folgendẹn Zeichen ausgedrückt.

Wachstum:-bedeutet negatives Wachstum, \pm wahrscheinlich positives Wachstum, $\dagger$ positives Wachstum von weniger als $1 / 5$ der Nährbodenfläche, \# positives Wachstum von $1 / 5-1 / 3$ derselben, \# positives Wachstum von 1/3-1/2 derselben und m positives Wachstum von der halben biz ganzer Nährbodenfläche.

Aussehen: ( $\rightarrow$ ) bedeutet, dass der Bazillenbelag trocken war, $( \pm)$ bedeutet äusserst geringer, dünner feuchter Belag, $(+)$ deutlich sichtbarer dünner feuchter Belag, (H) dünner lehmartiger feuchter Belag und (\#) dicker, lehmartiger feuchter Belag. 
Tabelle

Wachstum und Aussehen des BCG auf dem

\begin{tabular}{|c|c|c|c|}
\hline Nr. & Nährboden & & \\
\hline I & Glyzerinkartoffel mit alter Rindergalle & $\begin{array}{r}100 \% \\
50 \% \\
25 \% \\
10 \% \\
5 \%\end{array}$ & $\begin{array}{c}\text { Rindergalle } \\
\text { " } \\
" \\
" \\
"\end{array}$ \\
\hline II & Kartoffel mit alter Rindergalle & $\begin{array}{r}50 \% \\
25 \% \\
10 \% \\
5 \%\end{array}$ & $\begin{array}{c}\text { Rindergalle } \\
" " \\
"\end{array}$ \\
\hline III & Glyzerinkartoffel mit frischer Rindergalle & $\begin{array}{r}100 \% \\
50 \% \\
25 \% \\
10 \%\end{array}$ & $\begin{array}{c}\text { Rindergalle } \\
", \\
",\end{array}$ \\
\hline
\end{tabular}

Bemerkung: Wo.: Woche.

W.: Wachstum.

- negatives W.

\pm wahrscheinlich positives $W$.

+ positives $W$. von weniger als $1 / 5$ der Nährbodenfläche.

\# positives $W$. von $1 / 5-1 / 3$ derselben.

\# positives W. von 1/3-1/2 derselben.

H positives W. von halber bis ganzer Nährbodenfläche.

*1. Bazillenstamm wurde auf $5 \%$ Glyzeringallekartoffeln nach Calmette, und

2. Bazillenstamm auf Glyzerinbouillonkartoffeln immer in Intervallen von ungefähr 3 Wochen gezüchtet.

\section{Versuchsergebnisse.}

\section{Versuch I. Einfluss der Rindergalle.}

Wie man aus Tabelle 1 ersieht, wächst der erste Bazillenstamm schon von der 1. Woche an, wenn die Rindergalle alt ist, gleichviel ob dem Nährboden Glyzerin zugesetzt ist oder nicht und wievielfach die Galle verdünnt ist. Besonders auf Glyzerinkartoffeln mit Zusatz von Rindergalle in starker Verdünnung, d. h. von 5\% alter Rindergalle wächst er am besten. Beim Zusatz von frischer Rindergalle wächst er von der 1 . Woche an, nur wenn sie stark verdünnt ist. Ist sie nicht verdünnt oder nur schwach verdünnt, wächst er schwer oder beginnt erst nach Ablauf von mehreren Wochen zu wachsen. Was das Aussehen anbelangt, so wird im allgemeinen beim Zusatz von ungefähr $25 \%$ Rindergalle eine merkliche Feuchtigkeit beobachtet, wobei es keine grosse Rolle spielt, ob die Rindergalle frisch oder alt ist, ob dem 
I.

Kartoffelnährboden mit Rindergalle.

\begin{tabular}{|c|c|c|c|c|c|c|c|c|c|c|c|c|c|c|c|c|c|c|c|}
\hline \multicolumn{10}{|c|}{ *BCG von 1. Bazillenstamm } & \multicolumn{10}{|c|}{ *BCG von 2. Bazillenstamm } \\
\hline \multicolumn{2}{|c|}{ 1. Wo. } & \multicolumn{2}{|c|}{ 2. Wo. } & \multicolumn{2}{|c|}{ 3. Wo. } & \multicolumn{2}{|c|}{ 4. Wo. } & \multicolumn{2}{|c|}{ 5. Wo. } & \multicolumn{2}{|c|}{ 1. Wo. } & \multicolumn{2}{|c|}{ 2. Wo. } & \multicolumn{2}{|c|}{ 3. Wo. } & \multicolumn{2}{|c|}{ 4. Wo. } & \multicolumn{2}{|c|}{ 5. Wo. } \\
\hline W. & A. & W. & A. & W. & A. & W. & A. & W. & A. & W. & A. & $\mathbf{W}$ & A. & W. & A. & W. & A. & W. & A. \\
\hline $\begin{array}{l}+ \\
+ \\
H \\
+ \\
\text { 曲 }\end{array}$ & $\begin{array}{l}(+) \\
(+) \\
( \pm) \\
(+)\end{array}$ & $\begin{array}{l}+ \\
+ \\
+ \\
\text { H } \\
\text { WH }\end{array}$ & $\begin{array}{l}(+) \\
(+4) \\
( \pm) \\
(+)\end{array}$ & $\begin{array}{l}+ \\
+ \\
\text { 种 } \\
\text { 皿 } \\
\text { 曲 }\end{array}$ & $\begin{array}{l}(+) \\
(+) \\
(+1) \\
(+) \\
(+)\end{array}$ & $\begin{array}{l}+ \\
H \\
H \\
H+ \\
H+\end{array}$ & {$\left[\begin{array}{l}(\mathrm{H}) \\
(+) \\
(H) \\
(+H) \\
(+t)\end{array}\right.$} & $\begin{array}{l}H \\
H \\
H \\
\text { HI } \\
\text { HI } \\
\text { H }\end{array}$ & $\begin{array}{l}(\mathrm{H}) \\
(\mathrm{H}) \\
(\mathrm{H}) \\
(\mathrm{H}) \\
(\mathrm{H})\end{array}$ & $\begin{array}{l}\text { H } \\
H \\
H\end{array}$ & $\begin{array}{l}( \pm) \\
( \pm) \\
( \pm)\end{array}$ & $\begin{array}{l}H \\
\text { H } \\
\text { HH }\end{array}$ & $\begin{array}{l}( \pm) \\
( \pm) \\
( \pm)\end{array}$ & $\begin{array}{l}\text { H } \\
\text { HH } \\
\text { HH }\end{array}$ & $\left(\begin{array}{l}(-) \\
(+4)\end{array}\right.$ & $\begin{array}{l}\text { H } \\
\text { WH } \\
\text { HH }\end{array}$ & $\begin{array}{l}(-) \\
(-) \\
(+)\end{array}$ & $\begin{array}{l}\text { H } \\
\text { HW } \\
\text { HWH }\end{array}$ & $\begin{array}{l}(-) \\
(-) \\
(+)\end{array}$ \\
\hline $\begin{array}{l}+ \\
+ \\
+ \\
+\end{array}$ & $\begin{array}{l}(\mathrm{H}) \\
(\mathrm{H}) \\
(\mathrm{H}) \\
(\mathrm{H})\end{array}$ & $\begin{array}{l}+ \\
+ \\
+ \\
+\end{array}$ & $\begin{array}{l}(H) \\
(H) \\
(H) \\
(H)\end{array}$ & $\begin{array}{l}+ \\
+ \\
+ \\
+\end{array}$ & $\begin{array}{l}(+) \\
(H) \\
(H) \\
(H)\end{array}$ & $\begin{array}{l}+ \\
+ \\
+ \\
+\end{array}$ & $\begin{array}{l}(H) \\
(H) \\
(+4) \\
(H)\end{array}$ & $\begin{array}{l}+ \\
+ \\
+ \\
+\end{array}$ & $\left.\begin{array}{l}(+H) \\
(+H) \\
(H) \\
(H)\end{array}\right)$ & $\begin{array}{l}+ \\
+ \\
H \\
H\end{array}$ & $\left(\begin{array}{l}(-) \\
( \pm) \\
(-)\end{array}\right.$ & $\begin{array}{l}+ \\
H \\
H \\
H\end{array}$ & $\begin{array}{l}(-) \\
( \pm) \\
(-) \\
(-)\end{array}$ & $\begin{array}{l}H \\
H \\
H \\
H\end{array}$ & $\begin{array}{l}( \pm) \\
( \pm) \\
(-) \\
( \pm)\end{array}$ & $\begin{array}{l}H \\
H \\
H \\
H\end{array}$ & $\begin{array}{l}( \pm) \\
( \pm) \\
( \pm) \\
( \pm)\end{array}$ & $\begin{array}{l}H \\
H \\
H \\
H\end{array}$ & $\left(\begin{array}{l}( \pm) \\
( \pm)\end{array}\right.$ \\
\hline $\begin{array}{l}- \\
+ \\
H\end{array}$ & $\begin{array}{l}+1 \\
\pm\end{array}$ & $\begin{array}{c} \pm \\
+ \\
\text { H } \\
\text { HI }\end{array}$ & $\begin{array}{l}(-) \\
( \pm) \\
( \pm+1) \\
(-)\end{array}$ & $\begin{array}{l}\text { H } \\
\text { H } \\
\text { HI } \\
\text { WI }\end{array}$ & $\begin{array}{l}( \pm) \\
(\#) \\
(\#) \\
(-)\end{array}$ & 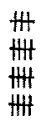 & $\begin{array}{l}( \pm) \\
(+) \\
(H) \\
( \pm)\end{array}$ & $\begin{array}{l}\text { H } \\
\text { Ht } \\
\text { Ht } \\
\text { Ht }\end{array}$ & $\begin{array}{l}( \pm) \\
(\mathrm{I}) \\
(\mathrm{H}) \\
( \pm)\end{array}$ & $\begin{array}{l}- \\
H \\
H\end{array}$ & $(-)$ & $\begin{array}{l}- \\
\pm \\
H \\
H\end{array}$ & $\left(\begin{array}{l}(-) \\
(-)\end{array}\right.$ & $\begin{array}{l}\overline{1} \\
\pm \\
H \\
H\end{array}$ & $(-)$ & $\begin{array}{l} \pm \\
+ \\
H \\
H\end{array}$ & $\begin{array}{l}(-) \\
( \pm) \\
(-) \\
(-)\end{array}$ & $\begin{array}{l} \pm \\
+ \\
+ \\
H\end{array}$ & $\begin{array}{l}(- \\
( \pm \\
(-\end{array}$ \\
\hline
\end{tabular}

\section{A. : Aussehen.}

(-) der Bazillenbelag trocken.

(士) äusserst geringer dünner feuchter Belag.

$(+)$ deutlich dünner feuchter Belag.

(H) dünner lehmartiger feuchter Belag.

(世\#) dicker lehmartiger feuchter Belag.

Nährboden Glyzerin zugesetzt ist oder nicht. Der 2. Bazillenstamm zeigt auf dem Kartoffelnährboden mit Zusatz von Rindergalle in verschiedenen Verdünnungen nur selten eine deutliche Feuchtigkeit auf dem Bazillenbelag. In bezug auf das Wachstum verhält er sich annähernd dem 1. Bazillenstamm ähnlich, d. h. auf Glyzerinkartoffeln mit Zusatz von Rindergalle in starker Verdünnung wächst er leicht, aber viel schwerer als der 1. Bazillenstamm. Aus diesem Versuche ist ersichtlich, dass, wenn man den BCG-Stamm dauernd auf 5\% Glyzerinkartoffeln mit Zusatz von vierfach verdünnter Rindergalle kultiviert, S-Kolonien am meisten erscheinen und auch das Wachstum am besten ist.

Versuch II. Einfluss gallensaurer Salze.

Die Verschiedenheit zwischen den 2 gebrauchten Bazillenstämmen ist dieselbe wie im Versuche I. Wie aus Tabelle 2 ersichtlich ist, wächst der 1. Bazillenstamm auf Glyzerinkartoffeln mit Zusatz von gallensauren Salzen am besten und zeigt eine deutliche Feuchtigkeit. Auf demselben Nährboden wächst der 2. Bazillenstamm verhältnismässig gut, zeigt aber nur eine 
Tabelle

Wachstum und Aussehen des BCG auf dem

\begin{tabular}{|c|c|c|c|c|c|c|}
\hline \multirow{3}{*}{ Nr. } & \multirow{3}{*}{ Nährboden } & \multirow{3}{*}{ - } & \multicolumn{4}{|c|}{$\mathrm{BCG}^{*}$ von } \\
\hline & & & \multicolumn{2}{|c|}{ 1. Wo. } & \multicolumn{2}{|c|}{ 2. Wo, } \\
\hline & & & $\mathrm{W}$. & A. & W. & A. \\
\hline $1, \mathrm{a}$ & Glyzerinkartoffel mit Natr. glycochol. & $\begin{array}{l}4,0 \% \\
2,0 \% \\
1,4 \% \\
0,8 \% \\
0,4 \% \\
0,1 \% \\
0,05 \%\end{array}$ & $\begin{array}{l}+ \\
+ \\
+ \\
+ \\
+ \\
+ \\
+4 \\
+\#\end{array}$ & $\begin{array}{c}( \pm) \\
(+) \\
(+) \\
(+m) \\
( \pm) \\
( \pm) \\
( \pm)\end{array}$ & $\begin{array}{l}+ \\
\# \\
\# \\
H \\
H \\
\text { WH } \\
\text { HI }\end{array}$ & $\begin{array}{l}( \pm) \\
(+) \\
(+) \\
(H) \\
(H) \\
( \pm) \\
( \pm)\end{array}$ \\
\hline 1, b & Kartoffel mit Natr. glycochol. & $\begin{array}{l}1,4 \% \\
0,4 \% \\
0,1 \% \\
0,05 \%\end{array}$ & $\begin{array}{l}4 \\
\pm \\
\pm\end{array}$ & $\begin{array}{l}(-) \\
(-) \\
(-)\end{array}$ & $\begin{array}{l}\text { m } \\
\pm \\
\pm\end{array}$ & $\begin{array}{l}(-) \\
(-) \\
(-)\end{array}$ \\
\hline $2, a$ & Glyzerinkartoffel mit Natr. taurochol. & $\begin{array}{l}4,0 \% \\
2,0 \% \\
0,8 \% \\
0,6 \% \\
0,4 \% \\
0,1 \% \\
0,05 \%\end{array}$ & $\begin{array}{l}+ \\
\pm \\
+ \\
+ \\
+ \\
\pm \\
+\end{array}$ & $\begin{array}{l}( \pm) \\
(-) \\
(+) \\
(+) \\
( \pm) \\
(-)\end{array}$ & $\begin{array}{l}+ \\
+ \\
+ \\
+ \\
+1 \\
+ \\
+\end{array}$ & $\begin{array}{l}( \pm) \\
(-) \\
(+i) \\
(+) \\
( \pm) \\
(-) \\
(-)\end{array}$ \\
\hline $2, \mathrm{~b}$ & Kartoffel mit Natr. taurochol. & $\begin{array}{l}0,8 \% \\
0,6 \% \\
0,4 \% \\
0,1 \% \\
0,05 \%\end{array}$ & $\begin{array}{l}+H \\
\frac{H}{H} \\
=\end{array}$ & $(+)$ & $\begin{array}{l}+\dot{f} \\
\text { +m } \\
= \\
-\end{array}$ & $\begin{array}{l}( \pm) \\
(+)\end{array}$ \\
\hline 3 & \multicolumn{2}{|l|}{$5 \%$ Glyzerinkartoffel. } & + & $(-)$ & $H$ & $(-)$ \\
\hline \multirow{4}{*}{4} & \multicolumn{2}{|c|}{$\begin{array}{l}\text { Glyzerinkartoffel mit Zusatz von } 4 \% \text { Natr. glycochol. u. } \\
0,8 \% \text { Natr. taurochol. }\end{array}$} & \# & $(+)$ & H & $(+)$ \\
\hline & \multicolumn{2}{|c|}{$\begin{array}{l}\text { Glyzerinkartoffel mit Zusatz von } 4 \% \text { Natr. glycochol., } \\
0,8 \% \text { Natr. taurochol., } 1,4 \% \text { Harnstoff u. } 0,9 \% \text { Kochsalz. }\end{array}$} & + & $( \pm)$ & + & $(+)$ \\
\hline & \multicolumn{2}{|c|}{$\begin{array}{l}\text { Glyzerinkartoffel mit Zusatz von } 1,4 \% \text { Natr. glycochol. u. } \\
0,6 \% \text { Natr. taurochol. }\end{array}$} & 册 & $(+)$ & 世 & $(+)$ \\
\hline & \multicolumn{2}{|c|}{$\begin{array}{l}\text { Kartoffel mit Zusatz von } 1,4 \% \text { Natr. glycochol. u. } 0,6 \% \\
\text { Natr. tautochol. }\end{array}$} & H & $(+)$ & $H$ & $|(+)|$ \\
\hline
\end{tabular}

äusserst geringe Feuchtigkeit. Wenn man das Vethalten des 1. Bazillenstammes genau betrachtet, so sieht man, dass er auf Kartoffelnährboden mit Zusatz von gallensauren Salzen allein, aber ohne Zusatz von Glyzerin im allgemeinen schwer oder in vielen Fällen gar nicht wächst. Auf Glyzerinkartoffeln mit Zusatz von Natr. glycochol. wächst er von der 1 . Woche an gut, besonders etheblich, wenn Natr. glycochol. in unter $0,4 \%$ Verdünnung beigegeben ist. Bezüglich des Aussehens zeigt er bei 2,0-0,8\% Konzentration eine deutliche Feuchtigkeit. Auf Glyzerinkartoffeln mit Zusatz von Natr. taurochol. wächst er im Vergleich mit seinem Wachstum beim Zusatz 
II.

Kartoffelnährboden mit gallensaurem Salz.

\begin{tabular}{|c|c|c|c|c|c|c|c|c|c|c|c|c|c|c|c|}
\hline \multicolumn{6}{|c|}{ 1. Bazillenstamm } & \multicolumn{10}{|c|}{$\mathrm{BCG}^{*}$ von 2. Bazillenstamm } \\
\hline \multicolumn{2}{|c|}{ 3. Wo. } & \multicolumn{2}{|c|}{ 4. Wo. } & \multicolumn{2}{|c|}{ 5. Wo. } & \multicolumn{2}{|c|}{ 1. Wo. } & \multicolumn{2}{|c|}{ 2. Wo. } & \multicolumn{2}{|c|}{ 3. Wo. } & \multicolumn{2}{|c|}{ 4. Wo. } & \multicolumn{2}{|c|}{ 5. Wo. } \\
\hline W. & A. & W. & A. & W. & A. & W. & A. & W. & A. & W. & A. & W. & A. & W. & A. \\
\hline 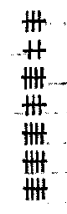 & $\begin{array}{c}(+) \\
(+H) \\
(H) \\
(+H) \\
(+) \\
( \pm) \\
(-)\end{array}$ & 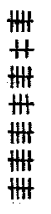 & $\begin{array}{c}(+) \\
(+H) \\
(+4) \\
(+H) \\
(+) \\
( \pm) \\
(-)\end{array}$ & 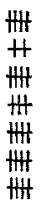 & $\begin{array}{c}(+) \\
(+H) \\
\text { (H) } \\
\text { (H) } \\
(+H) \\
( \pm) \\
(-)\end{array}$ & $\begin{array}{l} \pm \\
\frac{+}{H} \\
\text { H+ } \\
\text { W } \\
\text { WH } \\
\text { HH }\end{array}$ & $\begin{array}{c}(-) \\
(-) \\
( \pm) \\
( \pm) \\
( \pm) \\
( \pm)\end{array}$ & $\begin{array}{l} \pm \\
W \\
H \\
H \\
+W H \\
\text { WH }\end{array}$ & $\begin{array}{c}(-) \\
(-) \\
( \pm) \\
( \pm) \\
( \pm)\end{array}$ & 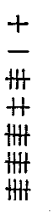 & $\begin{array}{l}(-) \\
(-) \\
( \pm) \\
(-) \\
( \pm)\end{array}$ & 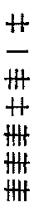 & $\begin{array}{c}(-) \\
(-) \\
( \pm) \\
(-) \\
(+)\end{array}$ & 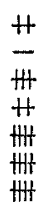 & $\begin{array}{l}(-) \\
(-) \\
( \pm) \\
( \pm) \\
( \pm) \\
(+)\end{array}$ \\
\hline $\begin{array}{l}\text { \# } \\
\pm \\
+\end{array}$ & $\begin{array}{l}(-) \\
(-) \\
(-)\end{array}$ & $\begin{array}{l}\text { \# } \\
+ \\
+\end{array}$ & $\begin{array}{l}( \pm) \\
(-) \\
(-)\end{array}$ & $\begin{array}{l}\frac{H}{+} \\
+\end{array}$ & $\begin{array}{c}(+) \\
(-) \\
(-)\end{array}$ & $\begin{array}{l}H \\
\pm \\
-\end{array}$ & $(-)$ & $\begin{array}{l}\text { \# } \\
\pm \\
-\end{array}$ & $(-)$ & $\begin{array}{l}+ \\
+ \\
-\end{array}$ & $(-)$ & $\begin{array}{l}+ \\
+ \\
-\end{array}$ & $(-)$ & $\begin{array}{l}+ \\
+ \\
-\end{array}$ & $(-)$ \\
\hline $\begin{array}{l}+ \\
+ \\
+ \\
\text { 锣 } \\
+ \\
+ \\
+\end{array}$ & $\begin{array}{l}( \pm) \\
(-) \\
(+H) \\
(+) \\
(+) \\
(-) \\
(-)\end{array}$ & $\begin{array}{l}+ \\
+ \\
+ \\
+ \\
+ \\
+ \\
+ \\
+\end{array}$ & $\begin{array}{c}(+) \\
(-) \\
(\text { II) } \\
(\text { II) } \\
(+) \\
(-) \\
(-)\end{array}$ & $\begin{array}{l}+ \\
+ \\
+ \\
\text { 冊 } \\
\text { 册 } \\
+ \\
+\end{array}$ & $\begin{array}{c}(+) \\
(+) \\
(+H) \\
(+) \\
(+) \\
( \pm) \\
(-)\end{array}$ & $\begin{array}{l}+ \\
+ \\
+ \\
+ \\
+ \\
+\end{array}$ & $\begin{array}{l}(-) \\
(-) \\
( \pm) \\
( \pm) \\
(-)\end{array}$ & $\begin{array}{l}- \\
+ \\
+ \\
+ \\
+ \\
+ \\
+\end{array}$ & $\begin{array}{l}(-) \\
(-) \\
( \pm) \\
( \pm) \\
( \pm) \\
(-)\end{array}$ & $\begin{array}{l}- \\
+ \\
+ \\
+ \\
+ \\
+ \\
+\end{array}$ & $\begin{array}{l}(-) \\
(-) \\
( \pm) \\
( \pm) \\
( \pm) \\
(-)\end{array}$ & $\begin{array}{l}- \\
+ \\
H \\
H \\
+ \\
+ \\
+\end{array}$ & $\begin{array}{c}(-) \\
(-) \\
( \pm) \\
(+) \\
( \pm) \\
(-)\end{array}$ & $\begin{array}{l}- \\
+ \\
H \\
+ \\
+ \\
+ \\
+\end{array}$ & $\begin{array}{l}(-) \\
(-) \\
( \pm) \\
(+) \\
( \pm) \\
(-)\end{array}$ \\
\hline $\begin{array}{l}+ \\
\stackrel{+}{+} \\
- \\
-\end{array}$ & $\begin{array}{l}( \pm) \\
(+)\end{array}$ & $\begin{array}{l}+ \\
\ddot{H} \\
= \\
-\end{array}$ & $\begin{array}{l}( \pm) \\
( \pm)\end{array}$ & $\begin{array}{l}+ \\
\ddot{H} \\
-\end{array}$ & $\begin{array}{c}(+) \\
( \pm)\end{array}$ & $\begin{array}{l}+ \\
+ \\
+ \\
+ \\
+\end{array}$ & $\begin{array}{l}(-) \\
(-) \\
(-) \\
(-)\end{array}$ & $\begin{array}{l}H \\
H \\
H \\
H\end{array}$ & $\begin{array}{l}(-) \\
(-) \\
(-)\end{array}$ & $\begin{array}{l}H \\
H \\
H \\
H \\
H\end{array}$ & $\begin{array}{l}(-) \\
(-) \\
(-) \\
(-)\end{array}$ & $\begin{array}{l}H \\
H \\
H \\
H\end{array}$ & $\begin{array}{l}(-) \\
(-) \\
(-) \\
(-)\end{array}$ & $\begin{array}{l}H \\
H \\
H \\
H\end{array}$ & $\begin{array}{l}(-) \\
(-) \\
(-)\end{array}$ \\
\hline$H$ & $(-)$ & $H$ & $( \pm)$ & $H$ & $( \pm)$ & H & $(-)$ & $H$ & $(-)$ & H & $(-)$ & m & $(-)$ & H & $(-)$ \\
\hline$\#$ & $(+)$ & $H$ & (H) & \# & (H) & & & & & & & & & & \\
\hline+ & $(+)$ & H & $(+)$ & m & (H) & & & & & & & & & & \\
\hline \# & $(+)$ & \# & $(+)$ & H & $(+)$ & & & & & & & & & & \\
\hline m & $(+)$ & \# & $(+)$ & m & $(+)$ & & & & & & & & & & \\
\hline
\end{tabular}

von Natr. glycochol. von der 1 . Woche an schon ziemlich schlecht; am besten wächst er in $0,8-0,4 \%$ Verdünnungen von Natr. taurochol., wobei auch eine deutliche Feuchtigkeit erkannt wird. Der 2. Bazillenstamm verhält sich annähernd gleich. Nach einer Mitteilung von Tagle und Kurth ${ }^{24)}$ enthält die normale Blasengalle des Menschen gallensaure Salze, welche $4 \%$ Natr. glycochol. und $0,8 \%$ Natr. taurochol. entsprechen. Wir haben gallensaure Salze von dieser Konzentration und das Gemisch, in dem ausser gallensauren Salzen Harnstoff und Kochsalz in dem Verhältnisse, wie sie in der Galle enthalten sind, d. h. 1,4\% bezw. 0,9\%, hergestellt. Ferner wurde ein 
Tabelle

Vergleich des BCG mit Tuberkelbazillen von

\begin{tabular}{l|c|c|c|c|c|c|}
\hline \multirow{2}{*}{ Nährboden } & \multicolumn{3}{|c|}{ Tuberkelbazillen von Typus humanus } \\
\cline { 2 - 6 } & \multicolumn{5}{|c|}{ Wachstum } \\
\cline { 2 - 6 } & 1. Wo. & 2. Wo. & 3. Wo. & 4. Wo. & 5. Wo. \\
\hline Glyzerinkartoffel mit 25\% iger Rindergalle. & - & - & - & - & - \\
\hline Kartoffel mit $25 \%$ iger Rindergalle. & - & - & - & - & - \\
\hline Glyzerinkartoffel mit 0,6\% igem Natr. tau rochol. & - & - & - & - & - \\
\hline Kartoffel mit 0,6\% igem Natr. taurochol. & - & - & - & - & - \\
\hline
\end{tabular}

* ebensowie Tab. I.

Nährmedium hergestellt, das aus $1,4 \%$ Natr. glycochol. $+0,6 \%$ Natr. taurochol. besteht. Züchtet man den 1. Bazillenstamm auf diesem Nährboden, so sieht man, dass, wie Tabelle II, 4 zeigt, das Wachstum des BCG verhältnismässig gut ist und auch eine Feuchtigkeit erkannt wird, wenn auch nicht gerade so, wie man erwartet hatte. Übrigens lässt sich das Vorhandensein des Einflusses gallensaurer Salze nicht übersehen. Diese zwei Bazillenstämme wachsen auf Glyzerinkartoffelnährboden von der 1. Woche an gut.

Versuch III. Vergleicb des BCG mit Tuberkelbaziillen von Typus bumanus auf dem Kartoffelnäbrboden.

In den Versuchen I und II wurde das Verhalten des BCG auf verschiedenen Nährböden beobachtet. Nun erhebt sich eine Frage, wie sich die Tuberkelbazillen verhalten. Wir haben mehrere Arten Nährboden, auf denen BCG in den Versuchen I und II das beste Wachstum oder eine deutliche Feuchtigkeit gezeigt hatte, hergestellt und auf diessen die Tuberkelbazillen von Typus humanus, welche in unserer Klinik fortwährend auf dem Eiernährboden von $\mathrm{Oka}$ und $\mathrm{Katakura}{ }^{25}$ ) kultiviert wurden, nach demselben Verfahren wie bei der Kultur von BCG gezüchtet. Aus diesem Versuche ergab sich, dass, wie aus Tabelle III ersichtlich ist, gat kein Wachstum erkannt wird. Im Kontrollversuche wächst BCG von der 1. Woche an gut, und der 1. Bazillenstamm zeigt eine deutliche Feuchtigkeit, was beim 2. Bazillenstamm nicht der Fall ist.

\section{Besprechung der Ergebnisse.}

Wenn man BCG auf Kartoffelnährboden mit Zusatz von Rindergalle kultiviert, so kann man im allgemeinen einen gleichmässig dicken, glänzend aschbraunen, lehmartigen, an Feuchtigkeit reichen Bazillenbelag erkennen. 
III.

Typus humanus auf dem Kartoffelnährboden.

\begin{tabular}{|c|c|c|c|c|c|c|c|c|c|c|c|c|c|c|c|c|c|c|c|}
\hline \multicolumn{10}{|c|}{ BCG* von 1. Bazillenstamm } & \multicolumn{10}{|c|}{ BCG* von 2. Bazillenstamm } \\
\hline \multicolumn{2}{|c|}{ 1. Wo. } & \multicolumn{2}{|c|}{ 2. Wo. } & \multicolumn{2}{|c|}{ 3. Wo. } & \multicolumn{2}{|c|}{ 4. Wo. } & \multicolumn{2}{|c|}{ 5. Wo. } & \multicolumn{2}{|c|}{ 1. Wo. } & \multicolumn{2}{|c|}{ 2. Wo. } & \multicolumn{2}{|c|}{ 3. Wo. } & \multicolumn{2}{|c|}{ 4. Wo. } & \multicolumn{2}{|c|}{ 5. Wo. } \\
\hline w. & A. & w. & A. & w. & A. & W. & A. & W. & A. & W. & A. & W. & A. & w. & A. & w. & A. & W. & A. \\
\hline 曲 & $(H)$ & 曲 & (\#) & HW & $(+1+)$ & WH & (姍) & 册 & {$[+\pi)$} & \# & $( \pm)$ & 世 & \pm () & \# & $(-)$ & H & $(-)$ & m & $(-)$ \\
\hline \# & (H) & m & (H) & H & (H) & \# & (\#) & H & (\#) & H & $(-)$ & H & $(-)$ & $H$ & $(-)$ & $H$ & -) & $H$ & $(-)$ \\
\hline \# & (H) & 册 & $(+)$ & 曲 & $(+)$ & 曲 & $(+)$ & 册 & $(+)$ & H & $(-)$ & $H$ & $(-)$ & $H$ & $(-)$ & $H$ & $(-)$ & $H$ & $(-)$ \\
\hline 1 & (H) & $\#$ & (卅) & $H$ & $(H)$ & $H$ & $(H)$ & H & $|(+)|$ & + & $(-)$ & $H$ & $(-)$ & $H$ & $(-)$ & $H$ & $|(-)|$ & $H$ & $(-)$ \\
\hline
\end{tabular}

Betrachtet man die Ergebnisse unserer Versuche, so scheint dabei die Konzentration der zugesetzten Rindergalle eine sehr bedeutende Rolle zu spielen. Beim Zusatz von 25\% Rindergalle wird die Erscheinung sog. S-Kolonie beobachtet, welche an Feuchtigkeit am reichsten ist. Betrachtet man das Wachstum des BCG, so ist es sehr verschieden, je nachdem die gebrauchte Rindergalle frisch oder relativ alt ist und Glyzerin dem Nährboden zugesetzt ist oder nicht, ganz von der Konzentration der Rindergalle zu schweigen. Beim Zusatz von alter Rindergalle wachsen die Bazillen im allgemeinen schon von der 1. Woche an leicht, während sie beim Zusatz von frischer Rindergalle schwer wachsen. Auf Glyzerinkartoffeln ist das Wachstum im Vergleich mit dem auf dem Nährboden ohne Zusatz von Glyzerin besser. Wenn die Rindergalle in starker Verdünnung ist, wachsen sie im allgemeinen leicht. Wie bereits oben erwähnt, wächst der 1. Bazillenstamm auf dem Nährboden mit Rindergallezusatz im Vergleich mit dem 2. leichter, was wahrscheinlich davon herrührt, dass jener der BCG-Stamm ist, der auf Kartoffelnährboden mit Rindergallezusatz kultiviert und an Rindergalle gewöhnt ist. Aus dem Gesagten könnte man sich vorstellen, dass frische Rindergalle von starker Konzentration eine hemmende Wirkung auf das Wachstum des BCG ausübt und dass eine bestimmte Konzentration der Rindergalle in einer gewissen Beziehung mit der Dissoziation des BCG steht.

Um den Einfluss der 2 Arten von gallensauren Salzen, Natr. glycochol. und Natr. taurochol., der Hauptbestandteile der Galle zu untersuchen, haben wir Versuche auf Nährböden mit Zusatz jeglicher Art von ihnen durchgeführt und festgestellt, dass die Konzentration der gallensauren Salze und der Zusatz von Glyzerin von grosser Bedeutung sind. Während BCG auf Nährboden ohne Zusatz von Glyzerin in vielen Fällen nicht wächst, wächst er auf Glyzerinkartoffeln mit Zusatz von Natr. glycochol. verhältnismässig 
gut, besonders gut, wenn Natr. glycochol. in unter $0,4 \%$ Verdünnung .ist, und zeigt eine deutliche Feuchtigkeit bei 2,0-0,8\%. Auf Glyzerinkartoffeln mit Zusatz von Natr. taurochol. wächst er im Vergleich mit seinem Wachstum auf eben geschilderten Nährboden schlimmer, bei 0,8-0,4\% Natr. taurochol. Konzentration sind Wachstum und Feuchtigkeit am deutlichsten. Gallensaure Salze, namentlich Natr. taurochol. haben eine wenn auch nicht grosse, hemmende Wirkung auf das Wachstum des BCG, aber sie üben sicherlich einen gewissen Einfluss auf die Dissoziation desselben aus. Wie oben erwähnt ist, enthält nach Tagle und Kurth die Blasengalle 4,0\% Natr. glycochol. und $0,8 \%$ Natr. taurochol. Wenn dem wirklich so ist, so findet man, dass die Konzentration gallensaurer Salze, wenn in unserem Versuche auf dem Nährboden mit Zusatz von gallensauren Salzen eine deutliche Feuchtigkeit, d. h. die Erscheinung der S-Form erkannt wird, gerade der Konzentration der in 2-4 fach verdünnter Galle enthaltenen gallensauren Salze entspricht. Auf dem Nährboden mit Rindergallezusatz sieht man, wie oben geschildert, eine deutliche Feuchtigkeit, wenn 50-25\% Rindergalle, nämlich 2-4 fach verdünnte Rindergalle zugesetzt ist. Als Ergebnis dieser Tatsache, darf man wohl sagen, dass Natr. glycochol. und Natr. taurochol. einen sehr grossen Einfluss auf die Erscheinung der S-Form von BCG ausüben. Wie schon in dèr Einleitung erwähnt, wurde seit alters das Veranlassungsmoment zur Dissoziation der Tuberkelbazillen untersucht, allein über die Beziehung zwischen der Erscheinung der S-Form von BCG und gallensauren Salzen liegen nur sehr wenige bemerkenswerte Mitteilungen vor. Nur Petroff berichtete, dass , $\mathrm{R}^{\mathrm{c}}$ - und , $\mathrm{S}^{\text {“c }}$-Form, die auf reinem Gentianaviolett'nährboden miteinander schwer zu unterscheiden sind, sich durch Zusatz von 0,25\% Natr. taurochol. leicht differenzieren lassen. Diese Mitteilung interessiert uns sehr, da sie mit unseren Versuchsergebnissen gewissermassen übereinstimmt. Auf dem Kartoffelnährboden, wo das Wachstum des BCG oder eine Feuchtigkeit am stärksten auftritt, wachsen die Tuberkelbazillen vom Typus humanus gar nicht. Ob diese Tatsache einfach darauf zurückzuführen sei, dass die Tuberkelbazillen von Typus humanus an Kartoffelnährboden mit Zusatz von Galle oder gallensauren Salzen nicht gewöhnt sind, oder auf den Unterschied der biologischen Eigenschaften der Tuberkelbazillen vom Typus humanus und derselben vom Typus bovinus, das ist eine Frage, die eine beșondere Untersuchung verlangt.

\section{Zusammenfassung.}

1) Kultiviert man BCG auf Kartoffelnährboden mit Zusatz von Rindergalle, so ist das Wachstum am besten, wenn dem Nährboden Glyzerin zugesetzt ist und alte Rindergalle in starker Verdünnung gebraucht wird. 
Was das Aussehen anbelangt, wird im allgemeinen bei ca. 25\% Rindergalle ein Bazillenbelag mit starker Feuchtigkeit beobachtet.

2). Auf dem Nährboden mit Zusatz von gallensauren Salzen und ohne Glyzerin wächst BCG schwer oder fast gar nicht. Auf Glyzerinkartoffeln mit Zusatz von Natr. glycochol. wächst er gut, besonders erheblich bei der Konzentration von unter $0,4 \%$, und bei $2,0-0,8 \%$ ist eine Feuchtigkeit am deutlichsten. Auf Glyzerinkartoffeln mit Zusatz von Natr. taurochol. wächst er im Vergleich mit seinem Wachstum auf denselben mit Zusatz von Natr. glycochol. schlechter, am besten wächst er bei der Konzentration von $0,8-0,4 \%$, und auch eine Feuchtigkeit ist innerhalb dieser Grenzen am deutlichsten.

3) Aus dieser Tatsache ist vorzustellen, dass Rindergalle, besonders ihre Hauptbestandteile, nämlich zwei Arten gallensaure Salze: Natr. glycochol. und Natr. taurochol., mit dem Wachstum und Aussehen der Kolonien des BCG, namentlich mit Erscheinung der S-Form desselben in einer engen Beziehung stehen muss.

\section{Literatur.}

(1) Baerthlein u. Toyoda, Zbl. Bakter., Abt. I, Ref. 1913, 57, 281.

(2) Gildemeister, Zbl. Bakter., Abt. I, Org., 1921, 86, 513.

(3) Petroff, Brauch u. Steenken, Proc. Soc. Exper. Biol. a. Med., 1927, 25, 14. Amer. Rev. Tbc., $1929,19,8$.

(4) Petroff, Steenkenu. Schnieder, Beitr. Klin. Tbc., 1930, 74, 499.

(5) Petroff u. Steenken, J. Exper. Med., 1930, 51, 832.

(6) Petroff, Klin. Wschr., 1931, 10, 247.

(7.) Kraus, Z. Immunit. forsch., 1929, 61, 454.

(8) Kraus u. Gerlach, Z. Immunit. Forsch., 1929, 62, 339.

(9) Tschechnowitzer, zit. nach Kraus (7).

(10) B. Lange, Dtsch. med. Wschr., 1930, 56, 927.

(11) Birkhau u, C. r. Soc. Biol., 1935, 119, 156, 370, 472.

(12) Roulet, Zbl. Tbk.-Forsch., 1937, 46, 273.

(13) Birkhaug, Amer. Rev. Tbc., 1933, 27, 6.

(14) Togunowa, Z. Tbk., 1933, 67, 36.

(15) Toda, Manshû Ig. Z., 1931, 15, 23.

(16) U ra be, Fukuoka Ik. Daig. Z., 1936, 29, 3008.

(17) Schaefer, C. r. Soc. Biol., 1935, 119, 59.

(18) Barglowski, Beitr. Klin. Tbk., 1936, 88, 139.

(19) Smithburn, J. Exper. Med., 1936, 63, 95.

(20) Oatway u. Steenken, Amer. Rev. Tbc, 1937, 135, 354.

(21) Tod a, Jicchi-Ika., 1937, 14, 333.

(22) Ot om o, Tôhoku Ig. Z., 1941, 29, 389.

(23) Calmette, Z. Tbk., 1928, 50, 124.

(24) Tagle u. Kurth, Klin. Wschr., 1937, 16, 1795.

(25) O ka, Nippon Rinsyo Kekkaku, 1940, 1, 59. 